\title{
El teatro como recurso didáctico para el aprendizaje de la Historia Medieval de España
}

\section{The Theater as didactic resource for learning Medieval History of Spain}

\author{
Javier López Rider ${ }^{1}$, Ricardo Córdoba de la Llave² \& M Trinidad Conde \\ González ${ }^{3}$
}

Fecha de recepción: 22/11/2020; Fecha de revisión:21/12/2020; Fecha de aceptación: 11/02/2021

Cómo citar este artículo:

López, J., Córdoba, R. \& Conde, M. T. (2021), El teatro como recurso didáctico para el aprendizaje de la Historia Medieval. Revista de Innovación y Buenas Prácticas Docentes, 10(1), 94-107.

\section{Autor de Correspondencia: lopezrider@uco.es}

\section{Resumen:}

El presente artículo recoge el desarrollo de un proyecto de innovación docente basado en el uso del teatro como recurso de aprendizaje para la enseñanza de la Historia, aplicado en la asignatura Historia Medieval de España, de $3^{\text {er }}$ curso del Grado de Historia de la Universidad de Córdoba, durante el curso 2019-2020. El proyecto se basa en la conocida idea de que lo que se aprende de memoria se olvida, pero lo que se trabaja se aprende para siempre. El diseño ha consistido en la realización de un trabajo de investigación de carácter grupal, sobre un hecho de relieve acaecido en los reinos cristianos de la España medieval, que los estudiantes debían entregar por escrito incluyendo cuanta información pudieran recopilar sobre el mismo, así como la elaboración de un guion en forma de diálogo que permitiera su puesta en escena. A continuación, to dos los grupos de trabajo desarrollaron la representación teatral del suceso estudiado en el aula universitaria, y algunos ante público infantil (alumnado de 3-5 años de la Escuela Infantil Margaritas) y ante público general (en el marco del programa del IMTUR Córdoba en Púrpura 2020). Con su desarrollo se ha conseguido que el estudiante aprenda Historia Medieval realizando un trabajo autónomo, colaborativo y motivador.

Palabras clave: Historia Medieval, Teatro, Investigación, España.

\begin{abstract}
:
This article lies with the development of a teaching innovation project based on the use of theater as a learning resource for the teaching of History. It was applied in the subject Medieval History of Spain, of the 3rd year of the Degree in History at the University of Córdoba, during the 2019-2020 academic year. The project is based on the well-known idea that what is learned by memory is forgotten, but what is worked on is learned forever. The design has consisted of carrying out a group research work on an important event that occurred in the Christian kingdoms of medieval Spain, which the students had to submit in writing including how much information they could collect about it, as well as the elaboration of a script in the form of dialogue that would allow its staging. Subsequently, all the working groups developed the theatrical representation of the event studied in the University classroom, and some of them before children (students 3-5 years old of the Margaritas Infant School) and before a general public (within the framework of Córdoba in Purple IMTUR Program 2020). With its development it has been achieved that the student learns Medieval History doing an autonomous, collaborative, and motivating work.
\end{abstract}

Key Words: Medieval History, Theater, Research, Spain.

\footnotetext{
${ }^{1}$ Universidad de Córdoba (España), lopezrider@uco.es, Código ORCID: 0000-0002-8412-921X.

2 Universidad de Córdoba (España), rcllave@uco.es, Código ORCID: 0000-0003-0186-7290.

${ }^{3}$ E.I. Margaritas, Córdoba(España), tcongon522@gmail.com.
} 


\section{INTRODUCCIÓN}

Desde hace tiempo el desarrollo de representaciones teatrales está considerado como un recurso apropiado para la enseñanza de la Historia (Oliva \& Torres, 1992; Vieites, 2014) y está siendo utilizado como metodología innovadora basada en la extendida idea de que, si lo que se aprende de memoria suele olvidarse con rapidez, lo que se trabaja de manera práctica, personal y directa se aprende para siempre (Navarro, 2007). Esta metodología, que ha sido contrastada con anterioridad en numerosos casos, ha sido aplicada en la Universidad de Córdobapara el estudio de la asignatura Historia Medieval de España, obligatoria de $3^{\mathrm{er}}$ curso del Grado de Historia, en una experiencia llevada a cabo durante el curso 2019-2020 con estudiantes de la décima promoción de dicho Grado (2017-2021).

El proyecto se ha planteado en una triple fase. En primer lugar, se parte de una búsqueda de información en la que se utilizan las técnicas de investigación propias de la disciplina histórica, por un grupo no superior a los seis estudiantes, sobre un hecho de relieve vinculado con la historia de los reinos cristianos hispánicos durante la Edad Media; investigación en la que los estudiantes se ven obligados a recurrir al examen de fuentes escritas coetáneas, bibliografía académica actual, documentación disponible en internet, materiales gráficos del período medieval, largometrajes, documentales o series de televisión, y cuantos materiales permitan obtener el perfecto conocimiento histórico del suceso elegido. En segundo término, y una vez establecidas las bases para el conocimiento académico del hecho (que debe haber sido lo suficientemente tratado por la historiografía como para hacer posible su conocimiento en profundidad y su reconstrucción detallada), el grupo de estudiantes hadebido presentar un trabajo escrito que incluye la elaboración de un guion, propio del grupo que trabajó sobre el tema o inspirado en la documentación consultada, pero que en todo caso permita hacer su representación teatral (Vaqueiro, 2014). Y, en tercer lugar, cada grupo ha efectuado su representación en forma de escena teatral tanto en el ámbito universitario como, tras su adaptación al público infantil, en la E.I. Margaritas (Álvarez \& Martín, 2016) e incluso, en algunos casos, ante la sociedad en general, como medio para exponer el relato histórico ante un público tanto conocedor de la materia como no especializado (Vieites, 2014).

\section{DESARROLLO DE LA EXPERIENCIA DE INNOVACIÓN}

En este apartado se señalan, en primer término, los objetivos a cumplir y las competencias que se querían desarrollar en el alumnado, para a continuación describir la experiencia de innovación que se presenta.

\subsection{Identificación de objetivos y competencias a adquirir}

La utilidad educativa del teatro hace de él una herramientaideal para que los estudiantes de cualquier ámbito del conocimiento, pero muy en particular de las Ciencias Sociales y Humanas, puedan abordar, de manera novedosa y eficaz, el estudio y comprensión de un hecho histórico de relieve, lo que permite completar el marco teórico de la lección magistral, habitualmente denso y poco atractivo, con una actividad mucho más práctica y dinámica que plantea como principales objetivos: 
a) Impulsar el trabajo autónomo, activo y colaborativo de los estudiantes, potenciando su motivación a la hora de interiorizar la información con su propia experiencia.

b) Mejorar la asimilación del conocimiento histórico de la España medieval gracias al recurso a una metodología docente atractiva e innovadora que permite adquirir las competencias previstas en la guía docente de la asignatura de un modo más directo, ameno y divertido.

c) Fomentar el feedbacky la relación de confianza mutua entre el profesor y el alumno/a, dando lugar a una relación más integradora y fluida de lo habitual.

Las competencias que el proyecto ha contribuido a desarrollar se cuentan entre aquellas, de carácter tanto específico como básico, incluidas en la Memoria del Grado de Historia de la Universidad de Córdoba y en la Guía Docente de la asignatura Historia Medieval de España del curso 2019-2020. En concreto, tener capacidad para transmitir información, ideas, problemas y soluciones a un público tanto especializado como no especializado (CB3), tener capacidad de trabajo en equipo (CB7), poseer las habilidades de aprendizaje necesarias para emprender estudios posteriores con un alto grado de autonomía (CB9) y tener capacidad para organizar, planificar y gestionar información de carácter general acerca de diversas sociedades y culturas (CE5).

Los principales objetivos que se han obtenido con el desarrollo del proyecto, desde el punto de vista de la etapa educativa de Infantil, han sido:

a) Propiciar el encuentro entre alumnado de Infantil y estudiantes de la Universidad.

b) Ofrecer a nuestro alumnado otra forma de ver la Historia, divertida y motivadora (Nicolás, 2011).

c) Poner en marcha procesos de investigación - acción colaborativa entre estudiantes, alumnado de infantil y profesorado universitario.

d) Capacitar al alumnado en el desarrollo de habilidades artísticas tan necesarias como las de carácter plástico y escénico.

\subsection{Desarrollo de la experiencia}

La experiencia de innovación docente fue realizada con estudiantes pertenecientes a la promoción 2017-2021 del Grado de Historia, se ha desarrollado a lo largo del primer cuatrimestre del curso académico 2019-2020 y ha estado articulada en tres fases distintas de trabajo:

\subsection{Fase 1. Investigación de base para elaboración del trabajo escrito y diseño del guion y representación escénica.}

Durante las cinco semanas iniciales del primer cuatrimestre, y en las horas de docencia semanal en grupo reducido, se presentó y explicó con detalle a los estudiantes en qué iba a consistir la actividad práctica de la asignatura, haciendo hincapié en que supondría un $40 \%$ del total de la nota en la evaluación final, al ser valorado con un $20 \%$ de la calificación final el trabajo escrito y con otro $20 \%$ la representación de la pieza teatral.

A continuación, se conformaron los grupos de trabajo, integrados por un mínimo de 4 y un máximo de 6 componentes. Seguidamente, y a partir de un listado de hechos de relieve ocurridos en los reinos cristianos de la España medieval, susceptibles de estudio y escenificación, of recido por el profesorado de la asignatura, se sortearon los temas propuestos y fueron elegidos nueve temas sobre los que se desarrollarían los 
trabajos y representaciones. A saber, el asesinato del rey Sancho II en el cerco de Zamora (1072), la jura tomada por el Cid al monarca Alfonso VI en la iglesia burgalesa de Santa Gadea (1072), el asesinato de Sancho IV de Navarra en el acantilado de Peñalén (1076), la defensa del castillo de Tarifa por Guzmán el Bueno ante los ejércitos benimerines (1294), la Farsa de Ávila protagonizada por la nobleza levantisca contra el rey Enrique IV (1468), la rebelión de los vecinos de Fuente Obejuna contra el comendador mayor de la Orden de Calatrava Fernán Gómez de Guzmán (1476), la captura del Gran Capitán durante la Guerra de Granada (1483), la proclamación como reina de Juana la Loca tras la muerte de su madre Isabel la Católica (1504) y el momento previo al fallecimiento del rey Fernando el Católico (1516).

A partir de que cada uno de estos hechos fuera asignado a un grupo de estudiantes, los integrantes del grupo debieron realizar un trabajo consistente en buscar tanto en bibliografía de carácter académico (manuales y monografías de Historia Medieval de España) como en obras de teatro, prosa y poseía, guiones de películas y series de TV, diálogos en series de animación, videojuegos o internet, información sobre el hecho a representar. Durante esta fase siempre contaron con el asesoramiento del profesorado de la asignatura, para guiarles acerca de dónde buscar y, sobre todo, para establecer criterios que les permitieran desechar datos poco fiables y errores, extraídos en numerosas ocasiones de recursos online.

\subsection{Fase 2. Preparación del guion y de la representación escénica.}

Durante el segundo tercio del cuatrimestre, entre la quinta y décima semana de clase, se desarrolló la segunda fase de la experiencia en la que, una vez estudiado el hecho histórico asignado a cada grupo, los integrantes llevaron a cabo su adaptación para la representación teatral, en escenas nunca superiores a los diez minutos de duración para hacerlas breves y dinámicas y permitir que contaran con un diálogo que hiciera posible su memorización por parte de los estudiantes (Mercer, 2004).

Los grupos dedicaron su tiempo a la redacción del trabajo escrito, poniendo en claro la importancia del hecho histórico a representar, confeccionando un diálogo acorde a la escena que se quería representar. Del trabajo escrito que cada grupo tenía que entregar, y que se integraba como un elemento más en la evaluación de la asignatura, formaba parte tanto la documentación obtenida sobre el tema como el guion que estaba previsto desarrollar en las correspondientes representaciones. Los guiones pudieron ser resultado tanto de un diálogo o escena localizada en las fuentes consultadas en la fase anterior 0 , más frecuentemente, de la elaboración propia del grupo.

Mientras se ajustaban y cerraban los diálogos, se adaptó la escena para no superar el citado tiempo de diez minutos, se llevó a cabo el reparto de personajes para comenzar los ensayos de manera inmediata y, con la colaboración de miembros del Grupo de Teatro de la Universidad de Córdoba y del profesorado de la E.I. Margaritas, los distintos grupos planificaron el atrezzo y la escenografía (Martín, 2014). El atrezzo fue conseguido mediante adquisición de simples disfraces basados en personajes de época medieval, y complementos de venta en cualquier tienda de disfraces (espadas, coronas, caballitos de juguete, etc.), mientras que en la E.I. Margaritas se llevaba a cabo el montaje de un escenario integrado por un telón que ocultara la entr ada y salida de personajes de la escena, una escena situada en un nivel elevado respecto al público infantil y un fondo con motivos que recordaban un paisaje medieval, a fin de dotar de veracidad a las representaciones para el público al que iban dirigidas. Estas escenas 
fueron objeto de repetidos ensayos por parte del grupo, a veces realizados en el espacio universitario, en otras ocasiones en el ámbito doméstico, donde se repitieron los diálogos para conseguir su aprendizaje, se ensayó revestirse del atuendo que luego se iba a emplear en la representación.

\subsection{Fase 3. Las representaciones teatrales.}

Durante las cinco últimas semanas del primer cuatrimestre, cada grupo entregó su trabajo escrito a través de la Plataforma Moodle, para su posterior evaluación, y llevó a cabo la representación teatral del hecho asignado durante las horas de docencia al grupo reducido, momento en el que esta actividad práctica fue evaluada por parte del profesorado como si se tratara de la exposición de un tema, teniendo en cuenta la idoneidad del diálogo y de la puesta en escena acorde con el vestuario, decorado y atrezzo utilizado, el buen reparto de los papeles entre los miembros del grupo, la soltura a la hora de hablar en público y de moverse por la escena, la capacidad de retener las frases memorizadas, la simpatía puesta de manifiesto en el acto.

El vocabulario y el control del nerviosismo se han tenido en cuenta, sobre todo, a la hora de adaptar el diálogo y el atrezzo a públicos diferentes, entre sus propios compañeros en clase, en el colegio infantil de las Margaritas o en el programa cultural de Córdoba en Púrpura. El esfuerzo y calidad de la puesta en escena ante públicos diferentes ha sido uno de los elementos importantes de cara a la evaluación final. Las representaciones fueron valoradas sobre la calificación global de la asignatura de la misma forma que anteriormente lo era la exposición oral del trabajo escrito, también llevada a cabo en clase de grupo reducido (Figura 1).

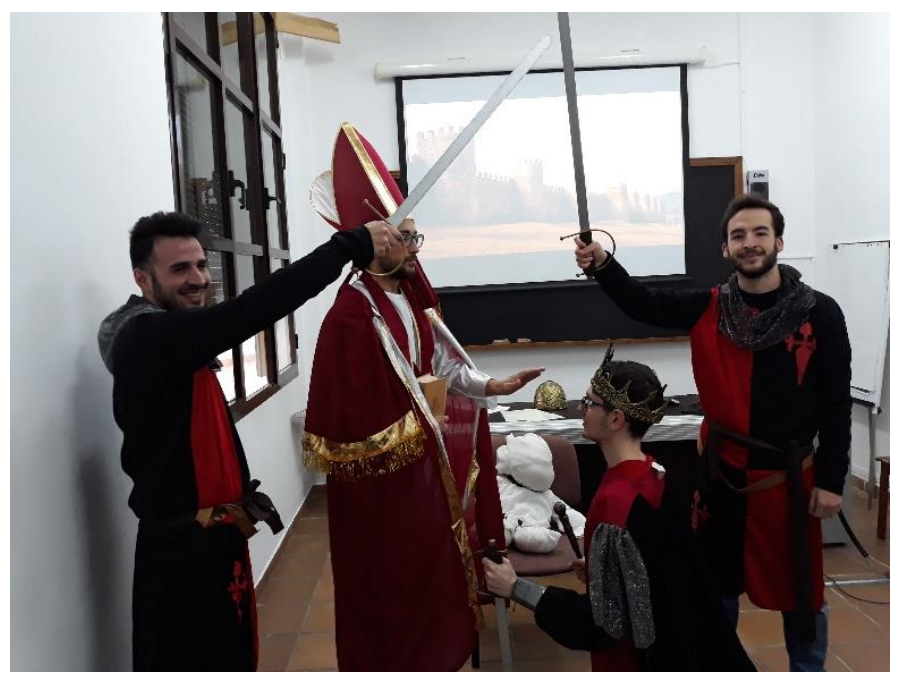

Figura 1. Representación de la Farsa de Ávila en clase de grupo reducido, en el aula 19 de la Facultad de Filosofía y Letras, el 11 de diciembre de 2019 (en escena, Jesús Berrios, Rafael Cot, Francisco J. Fernández y Rafael Hidalgo).

Aquellas obras mejor planteadas e interpretadas, más representativas del proyecto y que habían obtenido, por tanto, la mejor calificación en las representaciones efectuadas en las clases de grupo reducido, fueron elegidas para llevar a cabo una segunda representación en la E.I. Margaritas de la ciudad de Córdoba, ante un público infantil de 3-6 años de edad, en su mayor parte residente en la barriada de Las Moreras. Las obras fueron objeto de adaptación en su contenido, diálogo y atrezzo, parael público infantil, tanto a nivel de vocabulario, como en lo que se refiere a la eliminación de actos violentos o incorrectos o a la inclusión de otros que permitieran acercarse a niños de esas edades. El vestuario y atrezzo fue el mismo que se había empleado en las aulas 
universitarias, pero la actuación se llevó a efecto sobre el escenario preparado por las maestras del colegio (Figura 2).

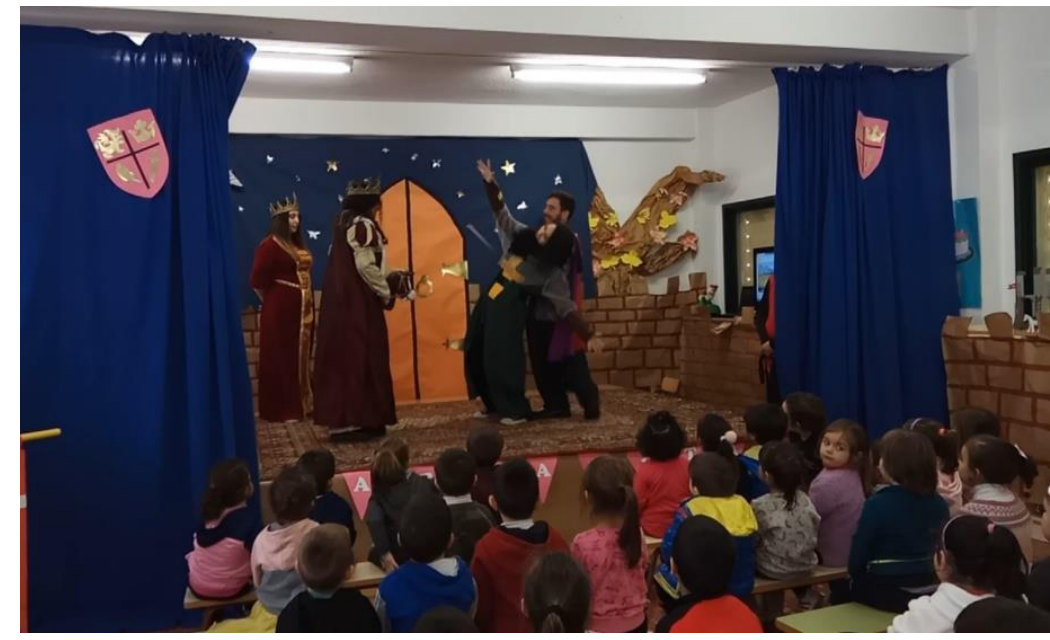

Figura 2. Representación del asesinato de Sancho Il en el cerco de Zamora, en la E.I. Margaritas de Córdoba, el 13 de diciembre de 2019 (en escena, Sara García, Esperanza Arroyo, Gloria de Arriba y Borja Rafael Herranz).

Para el desarrollo del proyecto en esta última fase se han efectuado, desde la E.I. Margaritas, las siguientes actividades. Se ha puesto en práctica un proyecto de Aprendizaje Basado en Proyectos (ABP) sobre la Historia Medieval de España, donde el alumnado de Infantil ha ido investigando y descubriendo cómo era la vida y costumbres de los habitantes de la Península lbérica en esta época de la Historia. En segundo término, y a lo largo de cuatro semanas, el alumnado de Infantil fue creando enseres, dibujos, vidrieras, vasijas y otros materiales, a fin de poder recrear el escenario ambientado en época medieval donde el alumnado de la Universidad tenía previsto hacer la representación teatral. Por último, al final del tercer trimestre, los estudiantes de la Universidad fueron pasando por la Escuela Infantil en grupo de cuatro o cinco, como ya se ha indicado, efectuando sus representaciones para la difusión del conocimiento histórico ante un público de edad muy corta y de situación social muy complicada, quizás escasamente motivado por los aspectos culturales, pero en cambio muy receptivo en actividades divertidas y motivadoras.

A inicios del segundo cuatrimestre, cuando ya había concluido el período de impartición de la asignatura en el curso 2019-20, fue aprovechada la puesta en marcha, por parte del Instituto Municipal de Turismo del Ayuntamiento de Córdoba (IMTUR), del Programa Cultural Córdoba en Púrpura, para que cuatro de los mejores grupos, que habían efectuado sus representaciones tanto en el aula de la Facultad como en la E.I. Margaritas, tuvieran ocasión de volver a representar sus escenas como un recurso para la difusión del conocimiento histórico ante un público de edades y situaciones sociales muy diversas (Nicolás, 2011, 103). La actividad fue realizada en el Centro de Recepción de Visitantes (CRV) de Córdoba, el sábado 15 de febrero de 2020, an te un público integrado por medio centenar de personas de todas las edades, y bajo el título de Recreaciones históricas de hechos destacados de la España cristiana en la Edad Media. Formaron parte de esta actividad las representaciones del asesinato del monarca Sancho II en el cerco de Zamora, la jura de Santa Gadea, la defensa del castillo de Tarifa por Guzmán el Bueno y la Farsa de Ávila (Figura 3). 


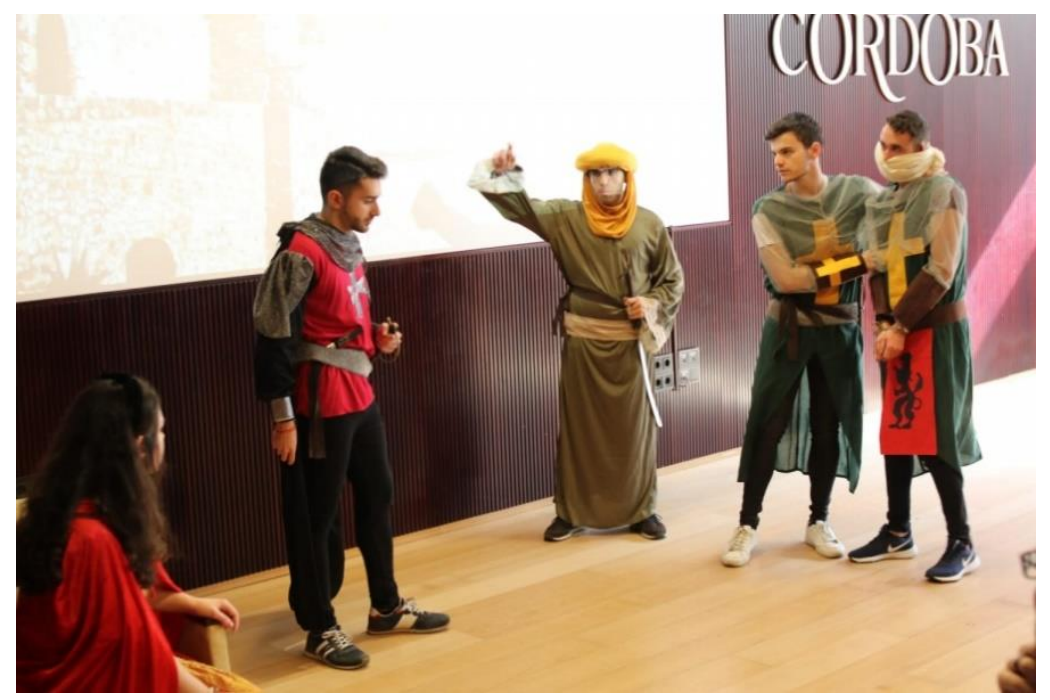

Figura 3. Representación de la defensa del castillo de Tarifa por Guzmán el Bueno, en el Centro de Recepción de Visitantes del Ayuntamiento de Córdoba, el 15 de febrero de 2020 (en escena, Álvaro Luque, Marco Mazón, Rafael Moreno, Noelia Priego y Jorge Reverte).

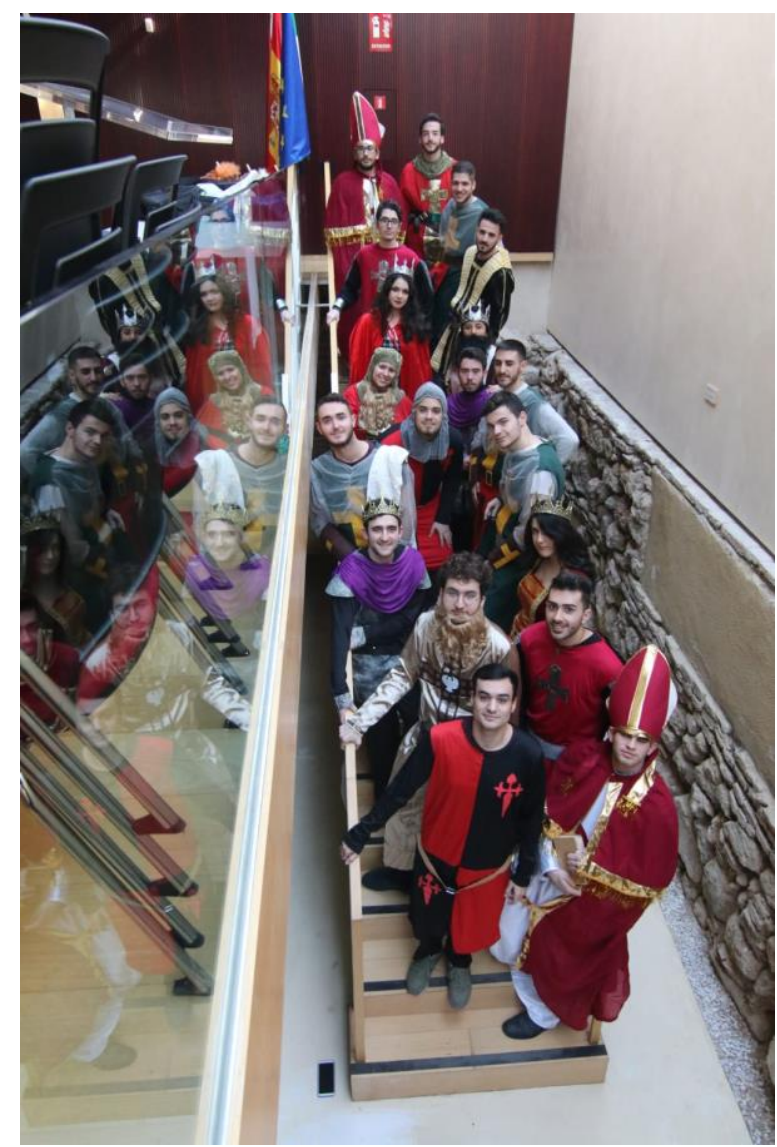

Figura 4. Grupo de estudiantes que participaron en las representaciones llevadas a cabo en el marco del Programa Córdoba en Púrpura (interior del Centro de Recepción de Visitantes). 


\section{RESULTADOS}

Se incluye en este apartado la valoración que los estudiantes de la asignatura han realizado del proyecto a través de las encuestas que se distribuyero n tras la finalización del cuatrimestre. Para la encuesta se realizaron siete preguntas, contestadas de manera anónima por los estudiantes, cuyos resultados están conectados con las distintas fases desarrolladas del proyecto que han sido expuestas en el apartado anterior.

Desde el punto de vista integral, se observa cómo los estudiantes han valorado muy bien la actividad como elemento evaluable en la nota final de la asignatura, otorgando la mayoría a este ítem la calificación de 5, la máxima posible (línea azul). También predomina una calificación alta en la utilidad que ha tenido, donde la mayoría opina que ha sido una experiencia gratificante mediante la que han aprendido historia medieval mientras se divertían (línea verde) (Figura 5).

Ligeramente baja de esa máxima calificación, la valoración sobre los criterios de evaluación aplicados en la actividad. Un porcentaje alto de estudiantes consideran que el trabajo escrito debería tener un papel menos predominante, bajando de ese $20 \%$ asignado. En cuanto a la dificultad que entraña toda la actividad práctica, se lleva como máximo un 2, es decir, no han encontrado gran complejidad a la hora de abordar el trabajo escrito y la representación teatral, lo que es un aspecto importante a tener en cuenta y demuestra el éxito del proyecto. Esto coincide con la puntuación mayor que recibe la valoración global, casi en su totalidad le dan la máxima nota posible a la actividad invitando a que se aplique más a menudo en el sistema universitario.

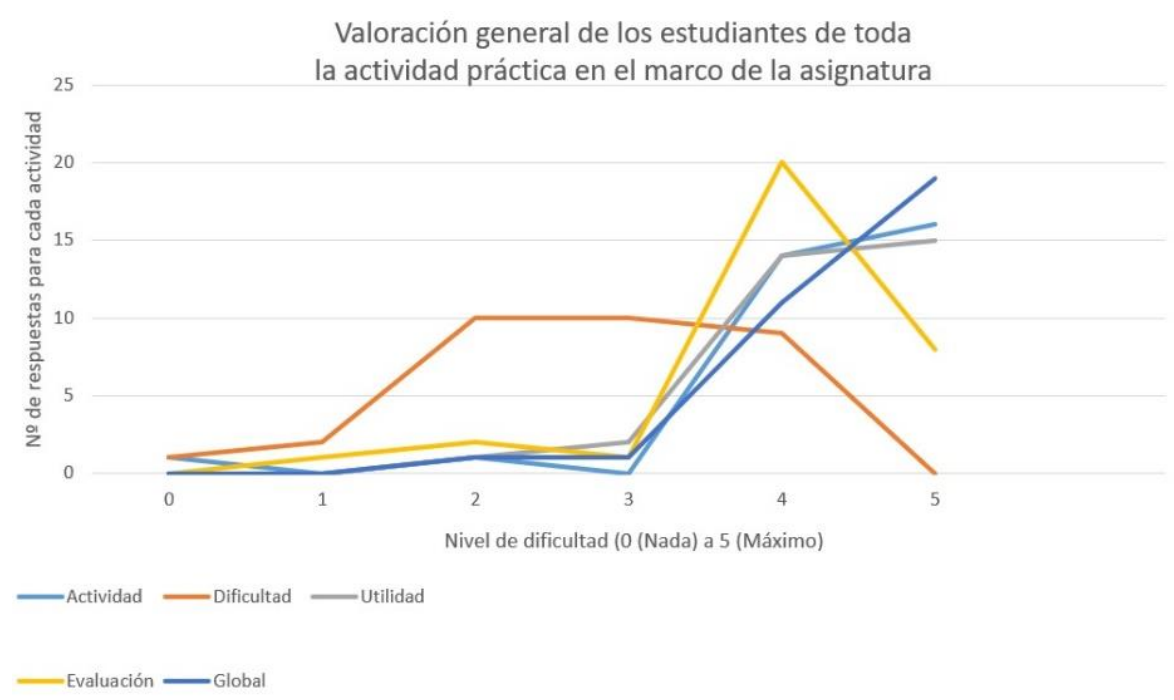

Figura 5. Resultados de la pregunta genérica sobre la actividad.

Si la encuesta se dirige hacia fases más concretas de la actividad práctica, se obtienen resultados más interpretables. Por ejemplo, si observamos los gráficos 2 y 3 , se puede identificar dónde han encontrado mayor dificultad a la hora de llevar a cabo la parte escrita (fases 1 y 2) o la representación teatral (fase 3). 
Respecto al trabajo escrito, el alumnado ha manifestado que coordinarse en grupo tiene una dificultad media-alta, al igual que la elaboración del guion. Sin embargo, más esfuerzo les ha llevado la búsqueda de información tanto de carácter bibliográfico y documental como para la preparación de la escenografía. Algo que sorprende es que no consideren compleja la redacción del trabajo escrito y cómo citar al pie o la propia bibliografía, porque normalmente suele ocasionar muchas dudas ante las diversas modalidades que hay para ello (Figura 6).



Figura 6. Resultados sobre la complejidad de las Fases 1-2 del trabajo.

En cuanto a la representación teatral, vuelve a repetirse de manera generalizada la dificultad de trabajar en grupo. Por ejemplo, el reparto de papeles y el tiempo ha sido valorado con un 4 sobre 5 , mientras que lo menos complejo para ellos fue la adecuación al guion de los temas de los hechos asignados. Una dificultad media-alta corresponde a los trabajos grupales que, a pesar de reconocer que tienen buena utilidad, no dejan de reiterar la complejidad que entraña el ponerse de acuerdo con sus compañeros y coordinar el rol que cada miembro del grupo debe desempeñar. En menor medida, las representaciones teatrales, tanto las llevadas a cabo en la clase universitaria como en las realizadas en el colegio infantil, suponen otro 4, con menos incidencia que el problema de la comunicación interna de los grupos de trabajo (Figura 7).

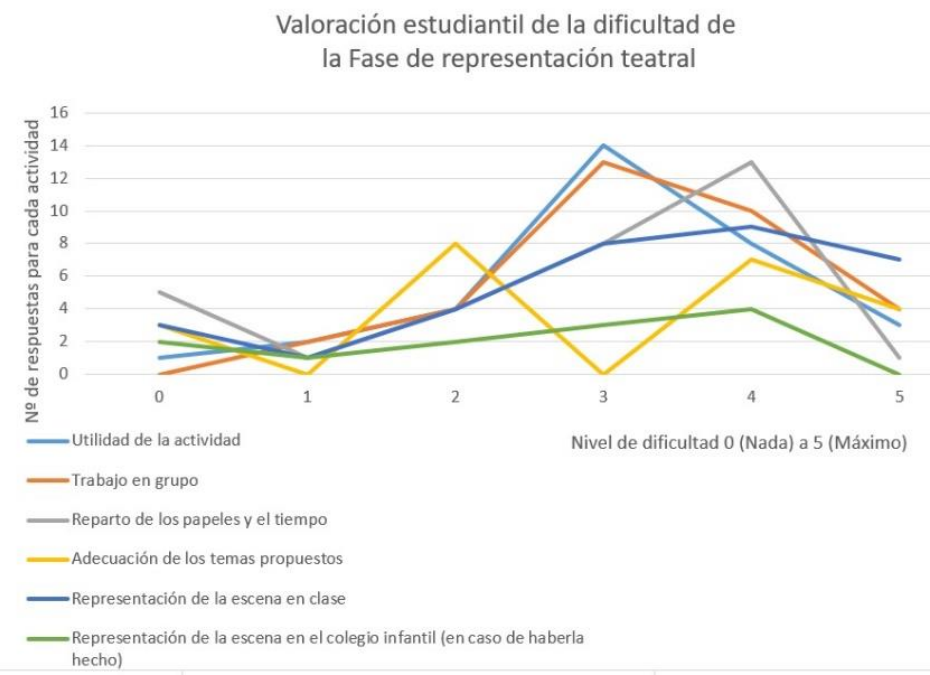

Figura 7. Resultados sobre la complejidad de la Fase 3 (representación de las escenas teatralizadas). 
Vinculadas con las mismas fases, se preguntó también sobre la valoración del nivel de aprendizaje adquirido con la actividad. Sobre la fase del trabajo escrito y la búsquedade información, casi toda la clase valora que han aprendido mucho trabajando en grupo, algo que coincide plenamente con la dificultad puesta de manifiesto en los gráficos anteriores. Es decir, a mayor dificultad, más esfuerzo y mejor aprendizaje. Lo mismo sucede con la búsquedade información pues, aunque supuso algo muy complejo ha permitido mejorar su aprendizaje autónomo a través del propio trabajo de cada estudiante, tanto a nivel particular como colaborativo con el resto de los compañeros de su grupo. Según parece, en la búsqueda bibliográfica y documental, así como en la redacción del trabajo, es donde menos consideran que han mejorado su proceso de aprendizaje, probablemente porque es un recurso utilizado de forma habitual en otras asignaturas del Grado de Historia (Figura 8).

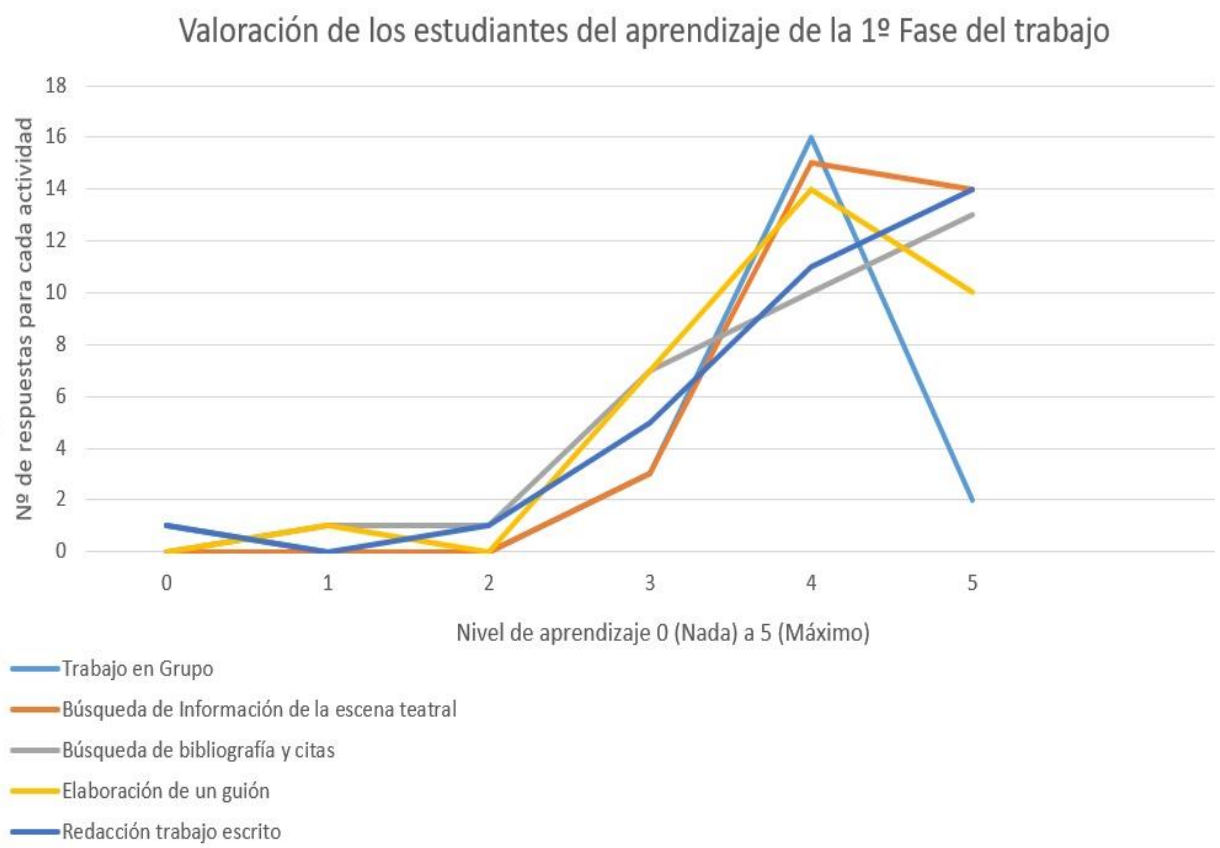

Figura 8. Resultados sobre el aprendizaje adquirido de la Fase 1. 
¿Qué sucede sobre la valoración de la representación teatral respecto del nivel de aprendizaje adquirido? Al tratarse de una actividad novedosay que nunca han tenido que desempeñar, los estudiantes la han aceptado con gran motivación y reconocen haber aprendido muchísimo. Si se fija la mirada en cada uno de los aspectos que se han detallado en el Figura 9, todos alcanzan, como mínimo, el 4 de valoración. Incluso, la utilidad de la actividad, el trabajo en grupo y la representación de la escena en clase o en el colegio infantil, supone la máxima calificación por igual. Eso tiene un significado muy positivo al determinar que el proyecto ha merecido la pena, pues la idea principal es que aprendan Historia Medieval de España. Sin embargo, aquí se refleja muchos otros aspectos que han aprendido y que, en un principio, no se contemplaban. Por ejemplo, ese trabajo en grupo o el reparto de papeles y tiempo, que tan difícil les ha sido afrontar, lo valoran con la máxima calificación, fruto de la enriquecedora experiencia obtenida. De hecho, a la pregunta sobre si la representación teatral efectuada en el colegio infantil les ha parecido una parte beneficiosa y positiva de la actividad, negativa o indiferente, el cien por cien de quienes participaron en ella seleccionaron la primera opción, mostrando un gran éxito entre los estudiantes

Valoración estudiantil del aprendizaje de

la Fase de representación teatral

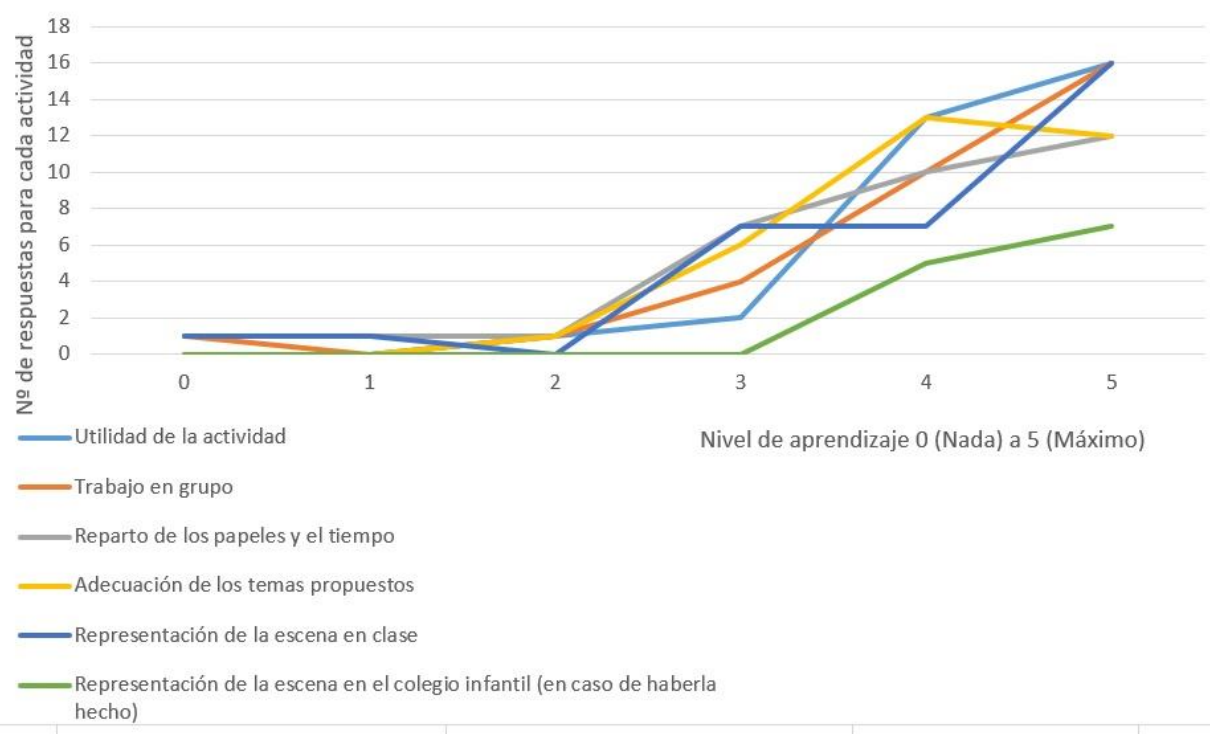

Figura 9. Resultados sobre el aprendizaje adquirido de la Fase 3.

A tenor de los resultados obtenidos en las encuestas, el proyecto ha tenido diversos aspectos de fortaleza. Por ejemplo, ha mejorado la autonomía del estudiante en su nivel de aprendizaje, facilitando la adquisición de las competencias recogidas en el Grado de Historia y en la guía docente de la asignatura. No solo ha mejorado su formación, sino que ha posibilitado que los estudiantes adquieran unos conocimientos teóricos de manera divertida, amena y potenciando el feedback con los propios compañeros de clase, el profesorado de la asignatura, del colegio infantil y los miembros del Grupo de Teatro de la Universidad de Córdoba. Al ser los protagonistas de su aprendizaje, han conseguido aprender, de manera activa, libre y eficiente, a buscar recursos y materiales, discernir qué fuentes son susceptibles de ser utilizadas y cuáles no, localizar información correctamente en la red y, sobre todo, elaborar un contexto histórico y un guion sobre un hecho importante del medievo hispano. La mayor parte de esas actividades, son novedosas y provocan la inquietud al estudiante que se motiva y esfuerza por realizarlas. 
Mismo rol juega la representación teatral, que es otro modo de expresarse oralmente, muy distinto de la tradicional exposición con unapresentación de diapositivas de powerpoint como apoyo, que la mayoría de estudiantes acaba no preparando bien ni dedicándole el tiempo necesario, por falta de interés y motivación. Por el contrario, con el teatro como herramienta de enseñanza, el alumnado tiene que ensayar las escenas, potenciasu interrelación y el trabajo en equipo, memorizar los diálogos permite aprender vocabulario, por ejemplo, del castellano antiguo, y datos relevantes de la historia sin ser conscientes de ello. Por supuesto, vestirse de época y convertirse en un personaje de aquel tiempo supone vivir una situación que no se olvide y aprender un conocimiento histórico con facilidad. Incluso la vocalización, el nerviosismo o cualquier otro problema que se presenta en una exposición clásica y tradicional, con la práctica del teatro se aprende mejor a controlar y superar.

Las consideraciones expresadas por las encuestas, resultan también útiles para señalar posibles mejoras de futuro. Por ejemplo, sus resultados indican que resulta esencial controlar más la comunicación y colaboración entre los miembros del grupo, algo que probablemente ha influido en que algunos grupos no hayan obtenido mejores calificaciones, al tener unos problemas en su funcionamiento interno que, en la mayoría de las ocasiones, no han sido trasladados al profesor. Otra alternativa es conformar grupos menos numerosos y proponer escenas más concretas y fijas, de manera que se pueda trabajar cómodamente de cara al reparto del tiempo y papeles entre los propios estudiantes. Algún que otro estudiante ha propuesto penalizar a aquellos miembros que no participen activamente o dificulten el buen desarrollo del proyecto grupal.

Por otra parte, conviene aportar a los estudiantes conocimientos adicionales antes de iniciar la búsqueda de información. Al principio, presentan muchos problemas porque no saben dónde acudir, ni si lo que han encontrado es realmente fiable para utilizarlo en la actividad propuesta. También es fundamental ayudarles a la elaboración del guion, proporcionándoles unas pautas en las primeras clases de grupo reducido que les pueda ser de gran utilidad, apoyándoles con ejemplos para que entiendan en qué consiste un guion y cómo redactarlo. En este sentido, cobra especial importancia que conozcan la realidad y su diferenciación con los mitos o leyendas medievales, de modo que puedan identificar lo que verdaderamente ocurrió, qué es fantasía y qué ha sido inventado para explicar el suceso. En último lugar, es necesario que se relacionen más con el castellano antiguo, por lo que se proporcionarían documentos medievales para su lectura de cara a una mejor memorización y comprensión de los diálogos.

Finalmente, de cara a la representación teatral, se propone como mejora llevar a cabo el aumento del número de ensayos realizados en el aula y otorgarles más tiempo para la representación. Para ello, se propone reforzar el papel de los miembros del Grupo de Teatro de la Universidad de Córdoba, que tendrían una función más activa y proporcionarían consejos y ayuda a los grupos conformados, mediante un enfoque distinto al del profesorado de la asignatura y una formación teatral específica. También resultaría muy beneficioso mejorar el atrezzo empleado en las representaciones, tanto a nivel de calidad del vestuario y complementos, como de la disponibilidad de una mayor variedad de elementos, lo que a su vez favorecería la posibilidad de proponer más temáticas con escenarios diferentes. 


\section{CONCLUSIONES}

Los resultados obtenidos con el desarrollo de este proyecto han sido altamente satisfactorios. El alumnado, casi en su totalidad, se ha implicado plenamente tanto en la parte escrita de la actividad como en la teatral. Aunque algunos grupos han destacado más que otros en una u otra faceta, el esfuerzo realizado, tiempo empleado y motivación demostrada por todos ellos, ponen de manifiesto el éxito rotundo de la aplicación del teatro como herramienta de enseñanza-aprendizaje en el sistema universitario.

Casi todos los estudiantes han confirmado una mejora de su conocimiento de los hechos de mayor relieve de la España medieval, lógicamente con mayor hincapié en el que han tenido que representar, al convertirse en personajes de la historia, como un monarca, un noble o una reina, y vivir una experiencia única que les ha permitido recordar mejor las fechas y lo ocurrido, de manera mucho más eficaz que si lo hubieran estudiado de memoria mediante el uso de un manual académico. No hay que olvidar la motivación que ha supuesto vivir la Edad Media durante unos minutos para potenciar el aprendizaje estudiantil, vinculado claramente con la autonomía del alumnado de cara a la preparación de los contenidos de la asignatura.

La fase 1 del trabajo resultó esencial para conseguir los objetivos previstos, puesto que permitió a los estudiantes acrecentar su conocimiento sobre el hecho histórico asignado, valorar los hechos de mayor relieve entre los vinculados a cada suceso, de forma que fueran seleccionados los más apropiados para ser representados, distinguir realidad y ficción del relato histórico en las fuentes investigadas y mejorar, en suma, su comprensión de la época medieval en la España cristiana.

Todos los grupos han llevado a cabo la representación de una escena, trabajada durante el cuatrimestre en las clases de grupo reducido, durante las cuatro últimas semanas del mismo; los más destacados de entre ellos, representaron la escena una segundavez, en la E.I. Margaritas antes pequeños de 3 a 6 años de edad, lo que generó un aprendizaje activo y positivo al realizar una actividad lúdica y recreativa y representar un reto para el alumnado universitario, el de entretener a los niños y niñas del colegio y divertir a las maestras, obteniendo el éxito de la representación proyectada (Sánchez, 2007; Zelaieta, Camino, Álvarez, \& Lasarte, 2014, 98).

El desarrollo del proyecto ha sido muy satisfactorio para el alumnado de la Escuela Infantil Margaritas. Una de las principales dificultades sentida por quienes han tenido que interpretar las escenas ha sido el nerviosismo de representar en castellano antiguo un guion que debían memorizar previamente, y hacerlo ante un público de edad muy corta para quien el vocabulario ha tenido que ser adaptado doblemente. Pese a que todos los participantes confirmaron que se trató de una gran experiencia, no es nada fácil transmitir fidedignamente el contenido de una escena y, mucho menos, convertirse en un personaje medieval para un grupo de niños/as de edades comprendidas entre los 3 y los 6 años, para quienes el vocabulario resultaba difícil de entender, pero aun así el dinamismo de las escenas les hizo seguir entusiasmados toda la representación (Tejerina, 1994).

Por su parte, la actuación de los cuatro mejores grupos en el marco del programa de divulgación científica Córdoba en Púrpura, organizado por el Instituto Municipal de Turismo del Ayuntamiento de Córdoba (IMTUR) durante el mes de febrero de 2020, supuso un elemento de motivación extra para el alumnado, al ver su trabajo premiado con una difusión a gran escala fuera del ámbito educativo y reconocido por un público muy diverso, que acudió expresamente a ver las actuaciones. Y tanto la adaptación al público infantil como a esta actuación en el CRV resultaron básicas como medio para difundir el conocimiento histórico ante la sociedad y desarrollar la competencia de 
transmitir información de carácter histórico ante un público tanto conocedor de la materia como no especializado (Vieites, 2014, 83-85).

Junto a esta formación para los estudiantes, el profesorado se ha beneficiado de la puesta en práctica, a nivel docente, de una metodología muy práctica y bien aceptada por el alumnado universitario. Si bien es verdad que hay que preparar una gran cantidad de material, el resultado lo justifica y hace que merezca la pena ante las calificaciones tan positivas obtenidas en la nota final con que los estudiantes de la asignatura han calificado al profesorado al cumplimentar las encuestas de la Universidad de Córdoba sobre la calidad docente. Por supuesto, dos ventajas añadidas son las relaciones que se forjan entre profesorado y alumnado universitario, así como de ambos colectivos con el profesorado y el alumnado del colegio infantil. De alguna manera, cabe concluir que ese y no otro ha sido el principal logro de esta actividad, la mejora de las relaciones académicas y personales entre profesorado y alumnado, el incremento del feedback mutuo, así como el grado de satisfacción alcanzado por los participantes, profesorado universitario, de educación infantil y estudiantes de la asignatura, todos de acuerdo en destacar lo enriquecedora que la experiencia había resultado.

\section{REFERENCIAS}

Álvarez Domínguez, P., \& Martín López, A. (2016). El teatro como herramientas didáctica en la enseñanza de la Historia de la Educación Contemporánea", Revista Digital de Investigación en Docencia Universitaria, 10, 41-51. Doi: http://dx.doi.org/10.19083/ridu.10.459.

García, A. (2004). Comunicación y expresión oral y escrita: la dramatización como recurso. Barcelona: Graó.

Martín, G. (2014). Interacción y motivación en el aula: enseñar desde el teatro. En A. M. Sainz García (2014), Arriba el Telón: enseñar Teatro y enseñar desde el teatro (pp. 4-19). Madrid: MECD.

Mercer, Á. (2004). Taller de teatro: cómo organizar un taller y una representación teatral. Barcelona: Alba ed.

Navarro, Ma ${ }^{a}$ R. (2007). Drama, Creatividad y Aprendizaje Vivencial: Algunas Aportaciones del Drama a la Educación Emocional. Cuestiones Pedagógicas. Revista de Ciencias de la Educación, 18, 163-174.

Nicolás, S. (2011). El teatro como recurso didáctico en la metodología CLIL: un enfoque competencial. Encuentro, 20, 102-108.

Oliva, C., \& Torres, F. (1992). Historia básica del arte escénico. Madrid: Cátedra.

Sánchez, Ma․ D. (2007). Teatro y educación. Revista RecreArte, 7. Recuperado de http://www.iacat.com/revista/recrearte/.

Tejerina, I. (1994). Dramatización y teatro infantil. Madrid: Siglo XXI.

Vaqueiro Romero, $M^{a}$ M. (2014). El teatro como recurso didáctico. En A. M. Sainz García (2014), Arriba el Telón: enseñar Teatro y enseñar desde el teatro (pp. 34-41). Madrid: MECD.

Vieites García, M. F. (2014). Educación teatral: una propuesta de sistematización. Teoría Educativa, 26, 77-101. Doi: http://dx.doi.org/10.14201/teoredu201426177101.

Zelaieta, E., Camino, I., Alvarez, A., \& Lasarte, G. (2013). Una experiencia teatral con estudiantes de Grado en Educación Infantil. Revista Electrónica 
Interuniversitaria de Formación del Profesorado, 16 (2), 93-103. http://dx.doi.org/10.6018/reifop.16.2.180671. 